OPEN ACCESS

Citation: Jun Wang, Qiang Ye, Chu Wang, Tong Zhang, Xusheng Shi, MajidKhayatnezhad,AbdulShakoor(2021) Palynological analysis of genus Geranium (Geraniaceae) and its systematic implications using scanning electron microscopy. Caryologia 74(3): 31-43. doi: 10.36253/caryologia-1109

Received: October 06, 2020

Accepted: July 21, 2021

Published: December 21, 2021

Copyright: (C) 2021 Jun Wang, Qiang Ye, Chu Wang, Tong Zhang, Xusheng Shi, Majid Khayatnezhad, Abdul Shakoor. This is an open access, peer-reviewed article published by Firenze University Press (http://www.fupress.com/caryologia) and distributed under the terms of the Creative Commons Attribution License, which permits unrestricted use, distribution, and reproduction in any medium, provided the original author and source are credited.

Data Availability Statement: All relevant data are within the paper and its Supporting Information files.

Competing Interests: The Author(s) declare(s) no conflict of interest.

\section{Palynological analysis of genus Geranium (Geraniaceae) and its systematic implications using scanning electron microscopy}

\author{
Jun Wang ${ }^{1,2,}$, Qiang Ye ${ }^{1}$, Chu Wang ${ }^{2}$, Tong Zhang ${ }^{2}$, Xusheng Shi ${ }^{2}$, Majid \\ KhayatnezHaD ${ }^{3}$, AbDul Shakoor 4,5 \\ ${ }^{1}$ School of Management, Harbin Institute of Technology; Heilongjiang Provice, China \\ ${ }^{2}$ Beijing Jinghang Computation and Communication Research Institute; BeiJing, China \\ ${ }^{3}$ Department of Environmental Sciences and Engineering, Ardabil Branch, Islamic Azad \\ University, Ardabil, Iran \\ ${ }^{4}$ College of Environment and Planning, Henan University, Kaifeng, 475004, Henan, China \\ ${ }^{5}$ Key Laboratory of Geospatial Technology for the Middle and Lower Yellow River \\ Regions, Ministry of Education, Kaifeng 475004, Henan, China \\ *Corresponding authors. E-mail: jxwxuwei@163.com; abdul_shakoor954@yahoo.com
}

\begin{abstract}
Pollen morphology of 23 species belonging to Geranium have been studied in details, which represent eight sections of two subgenera i.e., G. sect. Dissecta, Geranium, and Tuberosa of subgen. Geranium, Divaricata, Lucida, Ruberta and Trilopha of subgen. Robertium. These plant species were collected from different phytogeographical regions of Iran. The palynological investigation was done using scanning electron microscopy (SEM) techniques. Different palyno-morphological features have been observed, and the closely related species were distinguished. We used different multivariate statistical methods to reveal the species relationships. Ward clustering analyses have been done to check out the relationship among the species. The shapes of pollen grains were monad, radially symmetric, isopolar, apertures were tricolporate, and of spheroid, prolate-spheroid or sub-prolate classes. Three pollen types were recognized on the basis of differences in exine sculpturing pattern: reticulate-clavate, striaterugulate, reticulum cristatum with clavae. Observed differences were not of diagnostic importance in subgenera and sections level. The main objective of this study is to find distinguish pollen characters in the species of the genus Geranium and to elucidate their systematics importance.
\end{abstract}

Keywords: Geranium, pollen morphology, systematics, phytogeographical regions.

\section{RESEARCH HIGHLIGHTS}

- Pollen grains of the studied species were investigated by light and scanning electron microscope

- Using scanning electron microscopy techniques for the easy and quick identification of plant species

- Using micro-morphological (SEM) characters for the identification and species delimitations. 


\section{INTRODUCTION}

The Geranium L., is the largest genus of Geraniaceae and contains 325 species. Geranium species are distributed worldwide except in lowland tropical areas (Aedo 2017). Most of the species belong to subg. Geranium (Aedo 2001). Traditionally the genus Geranium was classified into three subgenera: 1- subg. Geranium, 2- subg. Erodioidea (Picard) Yeo, and 3- subg. Robertium (Picard) Rouy. According to Yeo (1984), the subg. Robertium primary diagnostic characteristics are carpel projection of fruit discharge. This subgenus has thirty species, and these are distributed in eight sections. The subgenus. Geranium, is the largest subgenus containing 300 species. These 300 species were in at least ten sections. The genus is characterized by a seed-ejection fruit type in which the explosive recurvature of the awn carries the mericarp upwards in an arc, throwing the seed out (Marcussen and Meseguer 2017). The family Geraniaceae Juss., is a worldwide family and comprising additionally five genera: Hypeocharis J. Remy (subfam. Hypseocharitoideae), Erodium L'Herit ex Aiton., Geranium L., Monsonia L., Pelargonium L'Herit. ex Aiton and California being successive sisters to Geranium L. (subfam. Geranioideae) (Fiz et al. 2008; Weng et al. 2014). These genera have a moderate number of species than Geranium and had a more narrow distribution.

The characteristic five-carpelled schizocarp Geranioideae fruit with a prominent central rostrum has a great taxonomic value in this genus. The Geranium shows four different fruit discharge methods: carpelprojection type, Erodium-type, seed-ejection type, and the inoperative type (Marcussen and Meseguer 2017). These methods have been considered in the delimitation of major groups within the Geranium. Marcussen and Meseguer (2017) pointed that phylogenetic relationships within Geranium and the evolution of the different seed discharge methods are still unknown. The new classification has shifted some of the studied taxa from subg. Robertium to subg. Geranium, while a third subgenus (Erodioidea) has been reduced to synonymy under subg. Geranium (Marcussen and Meseguer 2017).

The Geraniaceae is a eurypalynous family (Baser et al. 2016). The pollen morphological features of the family have a great importance in the discrimination at the generic level (Perveen and Gaiser 1999). The pollen grains are usually isopolar with radial symmetry; generally have oblate-spheroidal shape, rarely sub-oblate; three-lobed, tri-colporate, rarely colpate and the colpi are short; the sexine is thicker than nexine, and the tectum is reticulate with densely baculate or gemmate muri or striate (Perveen and Gaiser 1999).
Various workers described the pollen morphology of different species of Geraniaceae, species delimitation and species relationships in the genus Geranium L. Since Bortenschlager's (1967) preliminary study on the pollen morphology of the Geraniaceae, the pollen morphology of Monsonia (Verhoeven and Venter 1986), Sarcocaulon (Verhoeven and Venter 1988), and Erodium (Verhoeven andVenter 1987) of the southern African Geraniaceae has been investigated. Erodium pollen grains of the Middle East (EI-Oqlah 1983) and northwestern Europe have been studied (Stafford and Blackmore 1991). Stafford andBlackmore (1991) reviewed the pollen grains of Geranium species in north-western Europe. Pollen morphology of 35 taxa of the genus Geranium in Asia has been observed through light and scanning electron microscopy (SEM) by Park and Kim (1997). They found high variation in pollen grains size and exine sculpture (muri, lumina, excrescences distribution) among the examined taxa. Also, palynological characters were useful to establish the phylogeny within the genus. However, they are not helpful characters for separation at subgenera and sections. Perveen and Gaiser (1999) studied the pollen morphology of Pakistani Geraniaceae taxa and based on exine ornamentations and apertural type. They divided the pollen grains into three groups i.e., Erodium cicutariumGeranium himalayense, and Monsonia senegalensis-types. They observed that the pollen grains were mostly oblatespheroidal, rarely prolate-spheroidal or spheroidal, and often sub-oblate in the examined taxa. Shehata (2008) analyzed pollen grains of the Geraniaceae family from Egypt. The results showed that pollen morphological characters, i.e., pollen size, pollen shape, and aperture, were taxonomically significant at the generic level and up to some extent at the specific level. Ilcim et al. (2008) analyzed the morphological and palynological properties of G. tuberosum. Palynological investigation of 13 species of genus Geranium in Turkey by Deniz et al. (2013), did not reflect significant differences between the sections, except some differences in pollen grains of the subgenus Geranium. According to Deniz et al. (2013), all examined species' pollen grains were large, and exine ornamentation was reticulate-clavate; and the apertures were tri-colporate.

In Iran, the only study on pollen morphology of five Geranium species was assessed by Keshavarzi et al. (2016). There is no comprehensive palynological investigation of Geranium species in Iran. The present study aimed to use the pollen grains morphological features as a source of diagnostic characters to distinguish different Iranian Geranium species. 


\section{MATERIAL AND METHODS}

Totally 60 populations were collected and studied from 23 taxa of Geranium from different habitats in Iran to explore the pollen features (Table 1). Ten individuals of each location were studied and examined for three qualitative and eight quantitative features (Table 2). For scanning electron microscopy (SEM) examinations, pollen grains were not acetolyzed according to the method of Erdtman (1960). The pollens were suspended in a drop of water for a while and then directly transferred to a metallic stubby a fine pipette, and double-sided cello tape was used. And then the pollens were sputtered in a chamber coated with gold (Sputter Coater BALTEC, SCDOOS). Coating with gold by the physical vapor deposition method (PVD) was restricted to $100 \AA$. The SEM examination was carried out on a TESCAN microscope. Pollen sculptures were described according to previous terminology (Hesse et al. 2009).

The evaluated and measured characters of the species studied of Geranium are summarized in Tables II. To detect significant differences in the studied characters among the various studied species, an analysis of variance (ANOVA) was performed. For multivariate analysis, the mean of the quantitative characters was used. Principal Components Analysis (PCA) was performed among the species to determine palynological characters useful for separating the species. In order to group the species, cluster analysis using WARD (Minimum spherical variance) methods and PCA ordination plot were performed by PAST software (Hammer et al. 2001), using Euclidean and taxonomic distance among the species was calculated (Podani 2000).

The qualitative traits were coded as binary or multistate. Variables were systematized for multivariate statistical analysis. Average taxonomic distances and squared Euclidean distances were applied as dissimilarity coefficients in the pollen data cluster analysis. Image Tool Version 3.0 (http://ddsdx.uthscsa.edu/dig/itdesc.html) was used to carry out the required measurements.

\section{RESULTS}

\section{Infrageneric variation}

We showed that G. purpureum, G. robertianum and G. sylvaticum possess type I pollen grains. Sub-genus Geranium had type II pollens. Species, i.e., G. biuncinatum, G. mascatense and G. trilophum belonging to Trilopha section .showed the type III of pollen grains.

Factor analysis shows three factors explained more than $77 \%$ of the total observed variation in studied pol- len grains. The first factor revealed equatorial length, pollen shape importance. Aperture condition, colpus length, and exine thickness illustrated more than 44\% of the observed variation in the second factor. Principal component analysis (PCA) based on pollen grain qualitative and quantitative traits confirms the cluster analysis results by Ward's method.

\section{General pollen grain features}

The majority of Geranium species depicted prolatespheroidal pollen types. For instance, G. columbinum, $G$. collinum, G. sylvaticum, G. pratense, G. dissectum, G. linearilobum, G. tuberosum, G. kotschyi, G. platypetalum, G. gracile, G. ibericum, G. purpureum, G. pyrenaicum, G. robertianum, G. divaricatum, G. lucidum, and G. molle had prolate-spheroidal shape (Figures. 2 A1, B1, C1, D1; 3 E1, F1, G1; 4 M1, O1; 5 P1, Q1; 6T1, W1, Y1, Z1; 7 X1, U1). Spheroidal pollen types were observed in G. rotundifolium, G. pusillum, G. albanum (Figs. 4 L1, 3 H1, 4 N1). G. biuncinatum, G. mascatense, G. trilophum species had sub-prolate pollen morphology (Figures. 5 R1, S1; Figures. 7, U4-U6). We found that G. lucidum and G. robertianum had spheroidal-prolate pollen grains. While G. rotundifolium had spheroidal pollen shape (Table II, Figures 4 L1-L2). Our statistical and microscopy analyses depicted that G. platypetalum had the largest (Table II, Figure. 6 Z1, Z2) and G. pusillum possessed smallest pollen grains. (Table II, Figures 3 H1, H2).Our observations revealed that pollen grains were generally prolate-spheroidal except G. pusillum, G. rotundifolium, and G. albanum (Figures 3H1-H2, Figures 4 L1-L2; N1-N2). Mean polar axis length varied from $38.55 \mu \mathrm{m}$ (G. pusillum) to $104.88 \mu \mathrm{m}$ (G. platypetalum), while the mean of the equatorial axis length varied from $37.55 \mu \mathrm{m}$ (G. pusillum) to $105 \mu \mathrm{m}$ (G. platypetalum). The main colpus length varied from $12 \mu \mathrm{m}$ (G. molle) to $58 \mu \mathrm{m}$ (G. trilophum). P/E ratio differed from $0.89 \mu \mathrm{m}$ (G. albanum) to $1.5 \mu \mathrm{m}$ (G. trilophum). The main features of the investigated pollen grains are summarized in Table II. The basic ornamentation of the exine surface in the Geranium was reticulate-clavate, striate-rugulate, and reticulum cristatum with clavae. On the basis of differences in exine sculpturing pattern, the following 3 types are recognized: reticulate-clavate, striate-rugulate, reticulum cristatum with clavae. Most of the specimens belong to reticulum cristatum with clavae pattern.

\section{Type I: Geranium robertianum- type ("reticulate- clavate")}

Species: Geranium robertianum (Figure 5, P3; Figure 4, O3; Figure 3, E2)

Pollen class: Tricolporate 
Table 1. List of Geranium species in Iran their localities and voucher numbers.

\begin{tabular}{|c|c|c|c|}
\hline No & Section & Sp. & Locality \\
\hline 1 & Dissecta Yeo & G. dissectum L. & $\begin{array}{l}\text { Guilan, Lahijan } \\
\text { East Azerbaijan, Kaleybar, Cheshme Ali Akbar } \\
\text { East Azerbaijan, Kaleybar, Shoj-abad } \\
\text { Tehran, Damavand } \\
\text { Khorassan, Kashmar-Darvaneh }\end{array}$ \\
\hline 2 & Geranium & G. columbinum L. & $\begin{array}{l}\text { Guilan, Siahkal, Ezbaram; Guilan, Langerud, chaff; Guilan, Bandar-e Anzali; } \\
\text { Tehran, Damavand; Khorassan, Kashmar-darvaneh }\end{array}$ \\
\hline 3 & & G. rotundifolium $\mathrm{L}$. & $\begin{array}{l}\text { Tehran, Tuchal } \\
\text { Mazandaran, Kandovan-Siahbisheh } \\
\text { East Azerbaijan, Kaleybar, Shoj-abad }\end{array}$ \\
\hline 4 & & $\begin{array}{l}\text { G. collinum Stephan ex } \\
\text { Willdenow }\end{array}$ & $\begin{array}{l}\text { Mazandaran, Tonekabon-Jannat Rudbar } \\
\text { Hamedan, 20km s of Nahavand }\end{array}$ \\
\hline 5 & & G. sylvaticum $\mathrm{L}$. & East Azerbaijan, Kaleybar, Cheshme Ali Akbar \\
\hline 6 & & G. pratense L. & $\begin{array}{l}\text { East Azerbaijan, Kaleybar, Shoj-abad } \\
\text { Mazandaran, } 40 \mathrm{~km} \text { Tonekabon to Janat Abad }\end{array}$ \\
\hline 7 & Tuberosa (Boiss.) Reiche & $\begin{array}{l}\text { G. platypetalum Fisch. \& C. } \\
\text { A. Mey. }\end{array}$ & $\begin{array}{l}\text { East Azerbaijan, Kaleybar } \\
\text { Razavi Khorasan, Kashmar } \\
\text { Tehran, Damavand }\end{array}$ \\
\hline 8 & & G. ibericum Cav. & $\begin{array}{l}\text { Tehran, Damavand } \\
\text { Kordestan, Sanandaj } \\
\text { Khorassan, Kashmar-Darvaneh }\end{array}$ \\
\hline 9 & & G. gracile Ledeb. ex Nordm. & $\begin{array}{l}\text { Mazandaran, Noshahr, Kheyrud Kenar Forest } \\
\text { Kerman, Lalehzar, Baghabad }\end{array}$ \\
\hline 10 & & G. linearilobum DC. & $\begin{array}{l}\text { Tehran, Firuz kuh } \\
\text { Mazandaran, } 40 \mathrm{~km} \text { Tonekabon to janat abad }\end{array}$ \\
\hline 11 & & G. kotschyi Boiss. & $\begin{array}{l}\text { Alborz, Karaj- Qazvin } \\
\text { Tehran, Desin }\end{array}$ \\
\hline 12 & & G. tuberosum L. & East Azerbaijan, Kaleybar Cheshme Ali Akbar, Tehran, Tuchal \\
\hline 13 & Batrachioidea W.D.J. Koch & G. molle L. & $\begin{array}{l}\text { East Azerbaijan, Kaleybar, Shojabad } \\
\text { East Azerbaijan, Kaleybar, Cheshme Ali Akbar } \\
\text { Hamedan, 20km s of Nahavand }\end{array}$ \\
\hline 14 & & G. pyrenaicum Burm. f. & $\begin{array}{l}\text { East Azerbaijan, Kaleybar, roadside } \\
\text { East Azerbaijan, Kaleybar, Cheshme Ali akbar } \\
\text { East Azerbaijan, Kaleybar, Shojabad } \\
\text { East Azerbaijan, Babak fort } \\
\text { Tehran, Damavand }\end{array}$ \\
\hline 15 & & G. pusillum L. & $\begin{array}{l}\text { East Azerbaijan, Kaleybar, roadside } \\
\text { East Azerbaijan, Kaleybar Cheshme Ali Akbar } \\
\text { East Azerbaijan, Kaleybar, Shojabad } \\
\text { Hamedan, 20km s of Nahavand } \\
\text { Tehran, Damavand }\end{array}$ \\
\hline 16 & Ruberta Dumort. & G. purpureum Vill. & $\begin{array}{l}\text { East Azerbaijan, Kaleybar, Cheshme Ali Akbar } \\
\text { Guilan, Gole rodbar } \\
\text { Guilan, Gole rodbar, roadside } \\
\text { Guilan, Jirandeh } \\
\text { Khorassan, Kashmar-darvaneh } \\
\text { Kerman, Lalehzar, Baghabad }\end{array}$ \\
\hline 17 & & G. robertianum $\mathrm{L}$. & $\begin{array}{l}\text { Guilan, Gole rodbar } \\
\text { Esfahan, } 50 \text { km Delijan } \\
\text { Tehran, Damavand }\end{array}$ \\
\hline 18 & Divaricata Rouy & G. albanum M. Bieb. & $\begin{array}{l}\text { Guilan, Sangar, roadside } \\
\text { Guilan, Lahijan } \\
\text { Guilan, Jirandeh } \\
\text { Mazandaran, Siah bisheh to Chalus } \\
\text { Golestan, Ramian }\end{array}$ \\
\hline
\end{tabular}




\begin{tabular}{|c|c|c|c|}
\hline No & Section & Sp. & Locality \\
\hline & & & $\begin{array}{l}\text { Esfahan, } 50 \mathrm{~km} \text { delijan } \\
\text { Khorasan, Birjand } \\
\text { Tehran, Darakeh } \\
\text { Hamedan, } 20 \mathrm{~km} \text { s of Nahavand }\end{array}$ \\
\hline 19 & & G. divaricatum Ehrh. & $\begin{array}{l}\text { East Azerbaijan, Kaleybar } \\
\text { Tehran, Darband } \\
\text { Khorasan, Birjand } \\
\text { Tehran, Darakeh } \\
\text { Kerman, Lalehzar, Baghabad }\end{array}$ \\
\hline & Lucida R. Knuth & G. lucidum L. & East Azerbaijan, Kaleybar Cheshme Ali Akbar \\
\hline & Trilopha Yeo & G. trilophum Boiss. & $\begin{array}{l}\text { Hormozgan, Amani village, Kushk-e Nar Rural } \\
\text { Hamedan, 20km s of Nahavand }\end{array}$ \\
\hline 22 & & G. mascatense Boiss. & Hormozgan, Qeshm, Bakho Mountain \\
\hline 23 & & G. biuncinatum Kokwaro & Khuzestan, Shushtar- Masjed soleyman \\
\hline
\end{tabular}

Table. 2. Evaluated characters of pollen grains in Geranium species studied (values $M \pm S D \mu m$ ). M- Mean value; SD- Standard deviation. Aperture: At the same level =2, protruding = 1; Pollen shape: prolate-spheroidal =1, spheroidal= 2, subprolate=3; Exine ornamentation type: reticulate-clavate $=1$, striate-rugulose $=2$, reticulum cristatum with clavae $=3$

\begin{tabular}{|c|c|c|c|c|c|c|c|c|c|c|c|}
\hline Taxon & 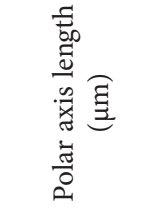 & 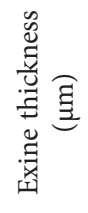 & 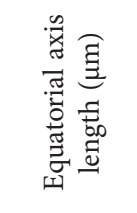 & 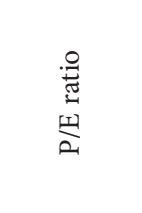 & 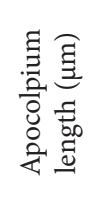 & 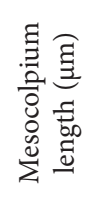 & 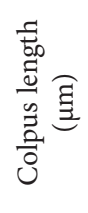 & 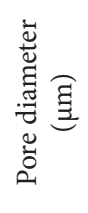 & 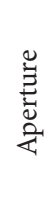 & 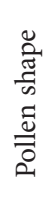 & 泀 \\
\hline G. dissectum & $55.67 \pm 2.1$ & 4.5 & $55.87 \pm 3.3$ & $\begin{array}{c}1.05 \pm 0.05 \\
\pm 0.03\end{array}$ & 23.67 & 35.66 & 36.44 & 6.87 & 1 & 1 & 3 \\
\hline G. columbinum & $62.76 \pm 3.4$ & 5.88 & $62.66 \pm 2.9$ & $1.00 \pm 0.03$ & 35.87 & 52.54 & 22 & 15 & 1 & 1 & 3 \\
\hline G. rotundifolium & $66.44 \pm 3.8$ & 5.87 & $65.54 \pm 2.3$ & $1.01 \pm 0.02$ & 37.22 & 35.77 & 25 & 8.5 & 1 & 2 & 3 \\
\hline G. collinum & $86.5 \pm 2.8$ & 5.34 & $77.32 \pm 4.8$ & $1.11 \pm 0.04$ & 48.55 & 34.33 & 55 & 12 & 1 & 1 & 3 \\
\hline G. platypetalum & $103.32 \pm 4.1$ & 4.45 & $101 \pm 2.7$ & $1.01 \pm 0.05$ & 68.87 & 54.77 & 33 & 24 & 1 & 1 & 3 \\
\hline G. sylvaticum & $78.44 \pm 2.9$ & 5.87 & $75.55 \pm 1.4$ & $1.04 \pm 0.04$ & 54.22 & 42.32 & 25 & 7 & 2 & 1 & 1 \\
\hline G. pretense & $75.55 \pm 3.7$ & 5.88 & $66.77 \pm 2.4$ & $1.06 \pm 0.02$ & 55.77 & 60.22 & 32 & 7 & 2 & 1 & 3 \\
\hline G. ibericum & $81.44 \pm 3.9$ & 4.34 & $78.33 \pm 3.6$ & $1.03 \pm 0.05$ & 47.22 & 54.55 & 28 & 13 & 2 & 1 & 3 \\
\hline G. gracile & $76.44 \pm 2.4$ & 5.45 & $74.56 \pm 2.9$ & $1.02 \pm 0.03$ & 37.55 & 53.77 & 27 & 16 & 1 & 1 & 3 \\
\hline G. linearilobum & $71.88 \pm 2.8$ & 5.65 & $61.55 \pm 4.2$ & $1.13 \pm 0.06$ & 38.43 & 38.34 & 39 & 14 & 1 & 1 & 3 \\
\hline G. kotschyi & $63.66 \pm 4.1$ & 5.8 & $59.55 \pm 4.5$ & $1.06 \pm 0.03$ & 42.66 & 35.77 & 39 & 11 & 1 & 1 & 3 \\
\hline G. tuberosum & $81.55 \pm 2.8$ & 5.3 & $75.66 \pm 2.2$ & $1.08 \pm 0.02$ & 48.77 & 46.44 & 33 & 12 & 1 & 1 & 3 \\
\hline G. molle & $49.44 \pm 1.6$ & 4.55 & $47.34 \pm 1.3$ & $1.04 \pm 0.04$ & 31 & 35 & 13 & 10 & 1 & 1 & 3 \\
\hline G. pyrenaicum & $64.22 \pm 2.8$ & 4.3 & $59.34 \pm 2.2$ & $1.08 \pm 0.05$ & 33 & 30 & 23 & 4 & 2 & 1 & 3 \\
\hline G. pusillum & $39.65 \pm 1.4$ & 3.7 & $38.22 \pm 5.3$ & $1.02 \pm 0.03$ & $26-27$ & 24 & 15.66 & 7.87 & 1 & 2 & 3 \\
\hline G. purpureum & $58.27 \pm 1.9$ & 4.2 & $54.55 \pm 2.5$ & $1.1 \pm 0.04$ & 37.85 & 29.01 & 27.68 & 7.4 & 2 & 1 & 1 \\
\hline G. robertianum & $61.76 \pm 4.1$ & 4.7 & $51.54 \pm 1.8$ & $1.02 \pm 0.02$ & 30 & $22-28$ & $21-22$ & $7-8$ & 2 & 1 & 1 \\
\hline G. albanum & $63.44 \pm 2.5$ & 5.45 & $66.34 \pm 2.6$ & $0.95 \pm 0.03$ & 38.76 & 39.44 & 26.33 & 14 & 1 & 2 & 3 \\
\hline G. divaricatum & $59.57 \pm 3.7$ & 4.55 & $51.66 \pm 3.9$ & $1.13 \pm 0.01$ & 33.66 & 35.33 & 32 & 9 & 1 & 1 & 3 \\
\hline G. lucidum & $58.55 \pm 5.2$ & 4.76 & $49.65 \pm 3.3$ & $1.09 \pm 0.04$ & 33.77 & 25.44 & 32 & 6 & 1 & 1 & 3 \\
\hline G. mascatense & $64.66 \pm 6.1$ & 5.88 & $60.33 \pm 2.9$ & $1.1 \pm 0.05$ & 23.77 & 47.55 & 50 & 14 & 1 & 3 & 2 \\
\hline G. trilophum & $65.44 \pm 2.6$ & 5.34 & $54.45 \pm 2.5$ & $1.2 \pm 0.03$ & 21.45 & 43.55 & 56 & 9 & 1 & 3 & 2 \\
\hline G. biuncinatum & $63.25 \pm 5.1$ & 4.35 & $63.76 \pm 7.9$ & $1.1 \pm 0.03$ & 22.65 & 45.98 & 59 & 12 & 1 & 3 & 2 \\
\hline
\end{tabular}




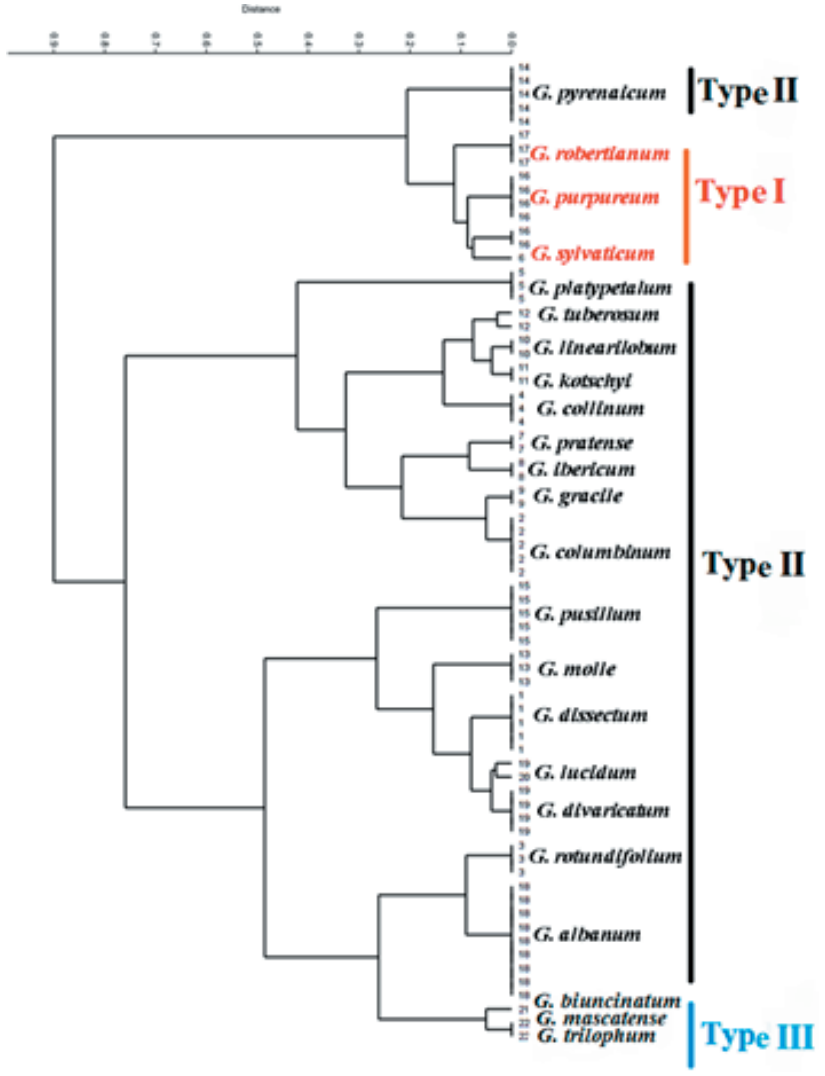

Figure 1. Ward clustering of Geranium species based on observed pollen data.

Shape: Prolate-spheroidal

Apertures: Ectoaperture-colpi small

Ornamentation: Tectum coarsely reticulate with clavae Measurements: polar length $(\mathrm{P})(56-75 \mu \mathrm{m})$, equatorial diameter $(\mathrm{E})(55-78 \mu \mathrm{m})$, colpi $(20-25 \mu \mathrm{m})$ in diameter. Mesocolpium (20-40 $\mu \mathrm{m})$. Apocolpium $(33-59 \mu \mathrm{m})$. Exine 4.9-5.9 $\mu \mathrm{m}$

Species included: G. robertianum, G. purpureum, G. sylvaticum

Type II: Geranium molle -type ("reticulum cristatum with clavae")

Species: G. molle (Figures 2, A3, B3, C3, D3; Figures 3, F3, G3, H3; Figures 4, L3, M3, N3; Figure 5, Q3; Figures 6, T3, W3, Y3, Z3; Figures 7, X3, U3 )

Pollen class: Tricolporate

Shape: Prolate- Spheroidal

Apertures: Ectoaperture - colpus short (approx. 1/5 to $1 / 8$ of polar axis)

Ornamentation: Tectum reticulum cristatum with clavae Measurements: Polar length (P) (38-105 $\mu \mathrm{m})$, Equatorial diameter (E) $(38-105 \mu \mathrm{m})$, colpi $(10-58 \mu \mathrm{m})$ in diameter. Mesocolpium $(22-67 \mu \mathrm{m})$. Apocolpium $(22-69 \mu \mathrm{m})$. Exine 3.7-5.8 $\mu \mathrm{m}$
Species included: G.gracile, G. ibericum, G. pyrenaicum, G. divaricatum, G. lucidum, and G. molle, G. pusillum ; G. columbinum, G. collinum, G. pratense, G. dissectum, G. linearilobum, G. tuberosum, G. kotschyi, G. platypetalum

Pollen type III: Erodium-type ("striate-rugulose")

(Figures. 5, R3, S3; Figures. 7, U4-U6)

Pollen class: Tricolporate

Shape: Sub- Prolate

Apertures: Ectoaperture-colpi large

Ornamentation: Tectum striate-rugulose

Measurements: Polar length $(\mathrm{P})(68-69 \mu \mathrm{m})$, Equatorial diameter (E) $(50-64 \mu \mathrm{m})$, colpi $(53-59 \mu \mathrm{m})$ in diameter.

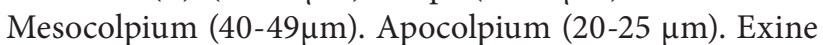
5.5-5.9 $\mu \mathrm{m}$

Species included: G. mascatense, G. trilophum, G. biuncinatum

\section{DISCUSSION}

Geranium species are relatively challenging to study due to the overlapping of morphological characters (Aedo and Pando 2017; Ji et al. 2020; Wang et al. 2020; Sun et al. 2021). Therefore, an attempt was carried out to investigate pollen grains of Geranium species by scanning electron microscopy. Our approach also included the usage of principal component analysis to verify our findings. The current study showed that Geranium species have eurypalynous pollens morphology. Present results corroborate with a previous study conducted on the Geraniaceae family (Baser et al. 2016). Most of the species showed prolate-spheroidal pollen types. This pattern of pollen type is previously reported in the Geraniance family (Baser et al. 2016). We observed monad, isopolar, radial symmetry, tricolporate, and short linear colpi pollen grains in Geranium species. Similar pollen types have been reported in Iran while working on five Geranium species (Keshavarzi et al. 2016). Our result recorded spheroidal-prolate pollen grains in G. lucidum and $G$. robertianum species. However, previously oblatespheroidal pollen grains were reported in G. lucidum, G. robertianum (Perveen and Gaisar 1999). We argue that such a difference in pollens is due to sampling site and habitat (Brodschneider et al. 2019). Annual or biennial species, i.e., G. lucidum, G. pusillum, G. molle, G. dissectum, G. rotundifolium showed the smaller pollen grains (from 39 to $66 \mu \mathrm{m}$ ). G. collinum, G. sylvaticum, G. pratense, G. platypetalum, G. gracile, and G. ibericum had larger $(63$ to $103 \mu \mathrm{m})$ pollen grains. Such variation in pollen grains has been described in the past (Aedo 2001; Aedo et al. 2007; Jurgens et al. 2012; Hao et al. 2020). 


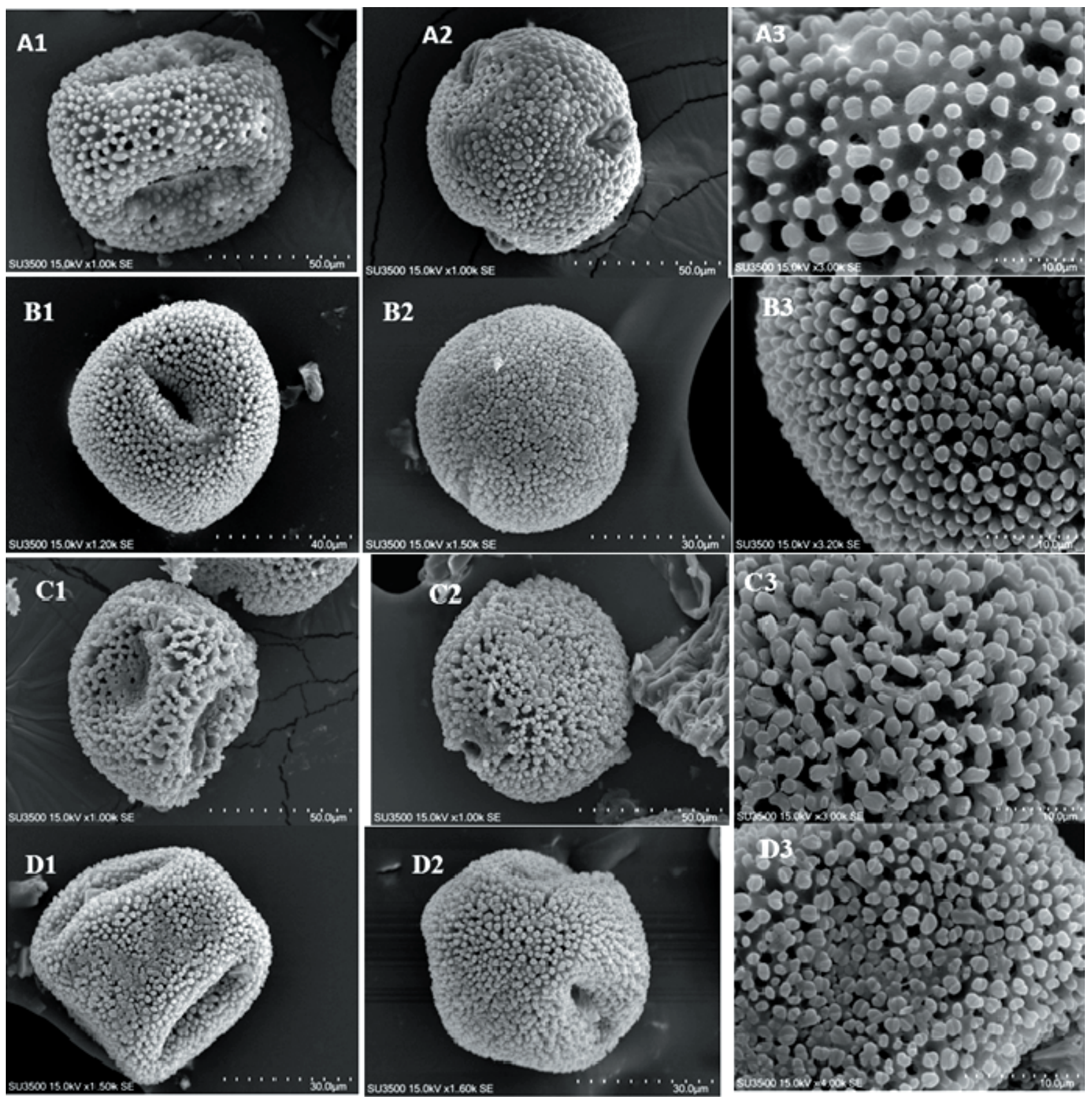

Figure 2. Pollen micrographs of Geranium species: A1-A3: G. collinum, B1-B3: G. columbinum, C1-C3: G. pratense, D1-D3: G. dissectum; A1, B1,C1, D1 - equatorial view; A2, B2, C2, D2 - polar view; A3, B3, C3, D3 -exine sculpture.

Clustering results revealed pollen grain type III in section Trilopha. Henceforth, type III pollen is the diagnostic feature to identify Trilopha Section.

Deniz et al. (2013) observed the exine ornamentation to be reticulate-clavate in Geranium taxa. While in the present study, exine ornamentations were reticulateclavate, striate-rugulose, reticulum cristatum with cla- vae. Most of the specimens belong to reticulum cristatum with clavae pattern. The pollen of all species of Geranium sect. Trilopha is of the Erodium-type (Aedo et al. 2016), which is congruent with the present findings. The striate ornamentation is considered a diagnostic feature to differentiate Trilopha section. This distinguishing feature may support the close relationship between G. sect. 

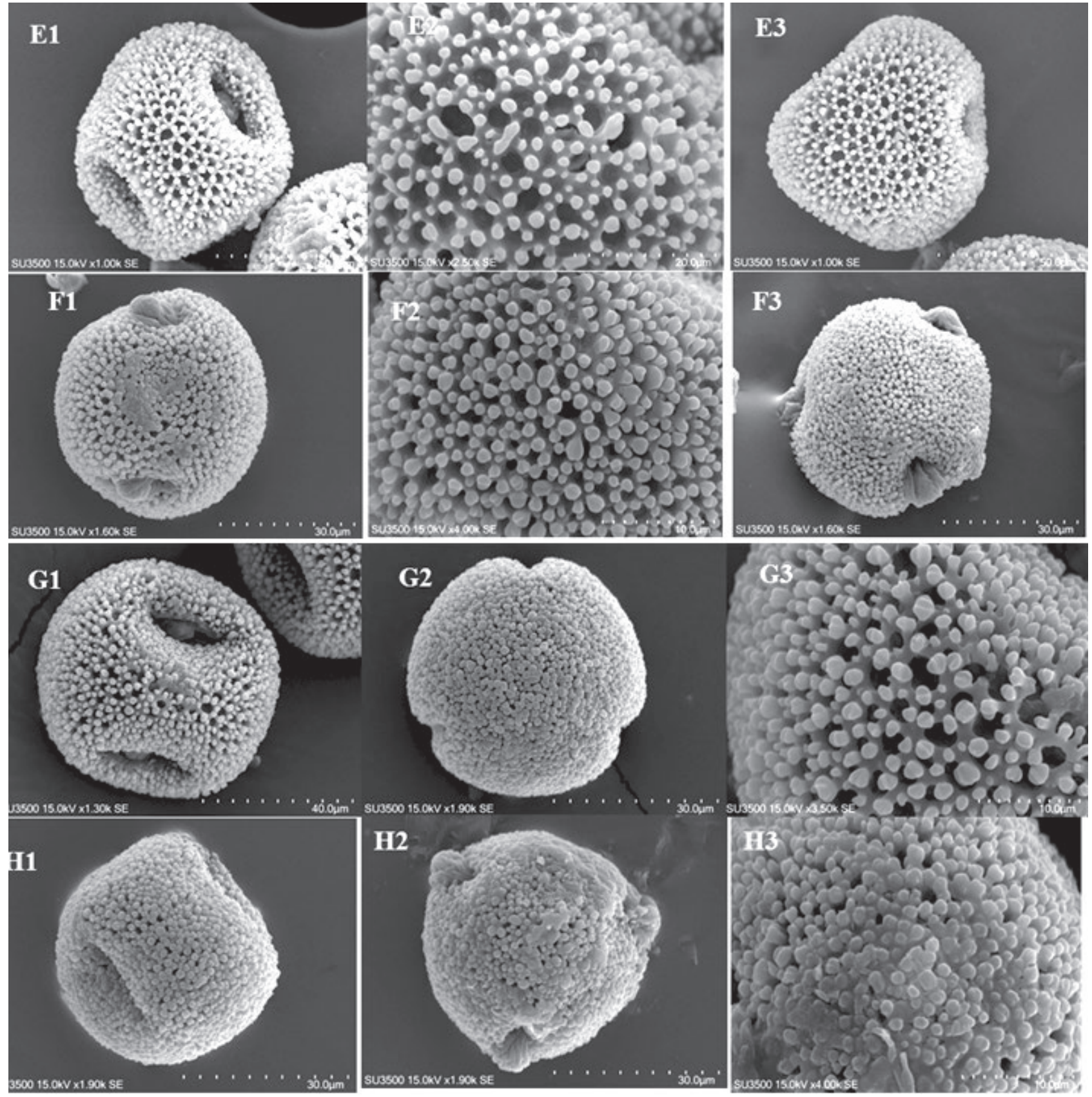

Figure 3. Pollen micrographs of Geranium species: E1-E3: G. sylvaticum, F1-F3: G. molle, G1-G3: G. pyrenaicum, H1-H3: G. pusillum; E1, F1, G1, H1 - equatorial view; E2, F2, G2, H2 - polar view; E3, F3, G3, H3 -exine sculpture.

Polyantha and G. sect. Trilopha.

The scanning electron microscopy (SEM) technique has a crucial role in observing the minute biological structure (Ullah et al. 2019). Several taxonomic and plant systematics questions are also addressed through the application of SEM. For instance, seed morphology in Caryophyllaceae was studied by scanning electron microscopy to identify Caryophyllaceae species correctly (Ullah et al. 2019; Zou et al. 2019). Besides this, detailed plant morphology, anatomy, and pollens in Spergula fallax and Spergula arvensis revealed the significance of SEM to resolve taxonomic complexity (Ullah et al. 2018). Authors developed identification key and distinguishing features of Spergula fallax and Spergula arvensis species 

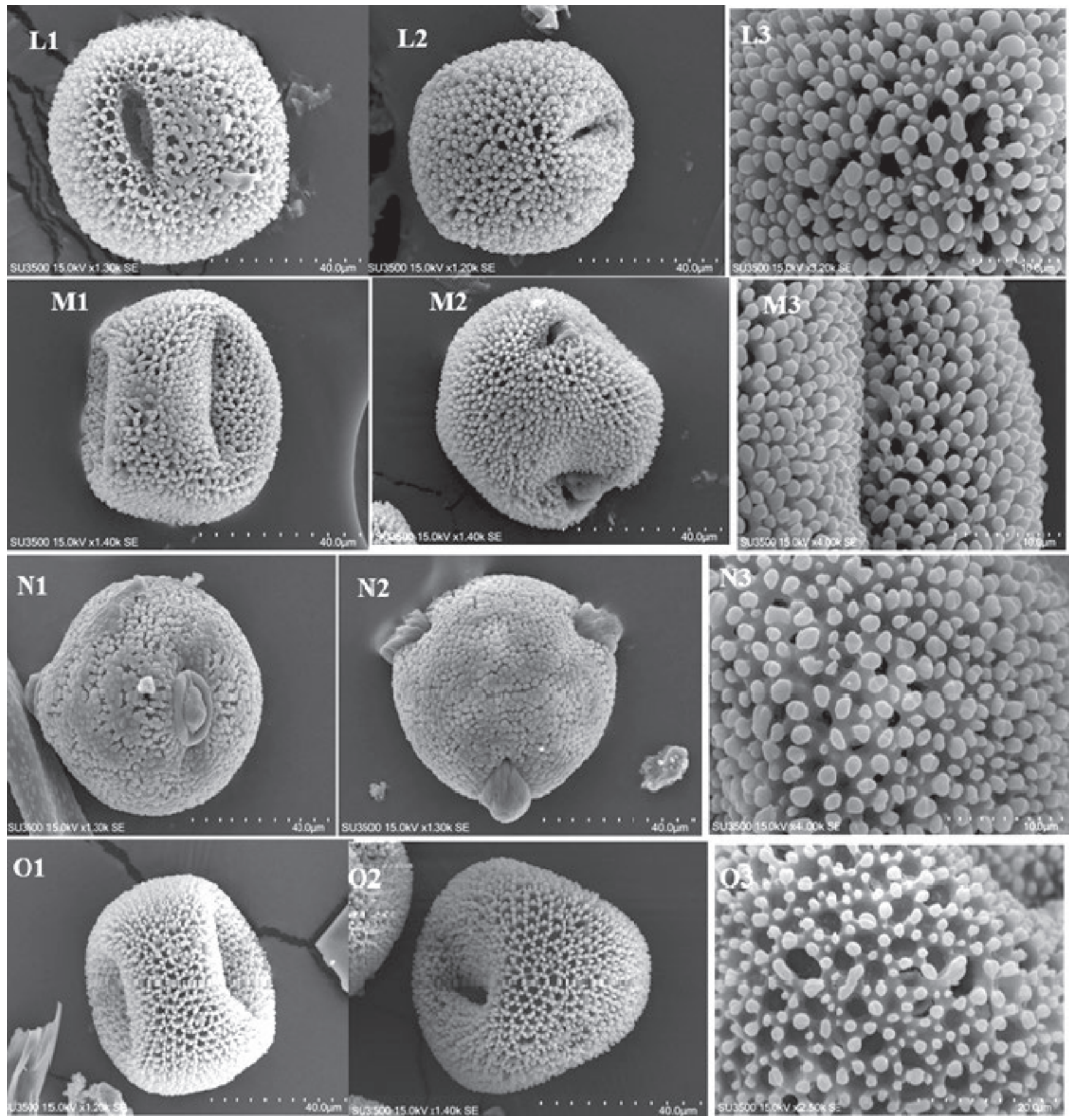

Figure 4. Pollen micrographs of Geranium species: L1-L3: G. rotundifollium, M1-M3: G. lucidum, N1-N3: G. albanum, O1-O3: G. purpureum ; L1, M1, N1, O1 - equatorial view; L2, M2, N2, O2 - polar view; L3, M3, N3, O3 -exine sculpture.

(Ullah et al. 2018). This clearly shows the utilization of new techniques to solve complex questions in plant systematics and biology (Pathan et al. 2010; Ullah et al. 2018; Ullah et al. 2019).

Cluster revealed groupings of G. uncinatum, G. mascatense, G. trilophum into the Trilopha section. Similar results at the section level was reported. Phylogenetic relationship based on chloroplast $(r b c \mathrm{~L}, \operatorname{trnL}-\operatorname{trnF})$ and nuclear (ITS) DNA sequences highlighted the grouping of G. uncinatum, G. mascatense into the Trilopha section (Marcussen and Meseguer 2017). The phylogenetic tree proved the Geraniaceae family as a monophyletic. 

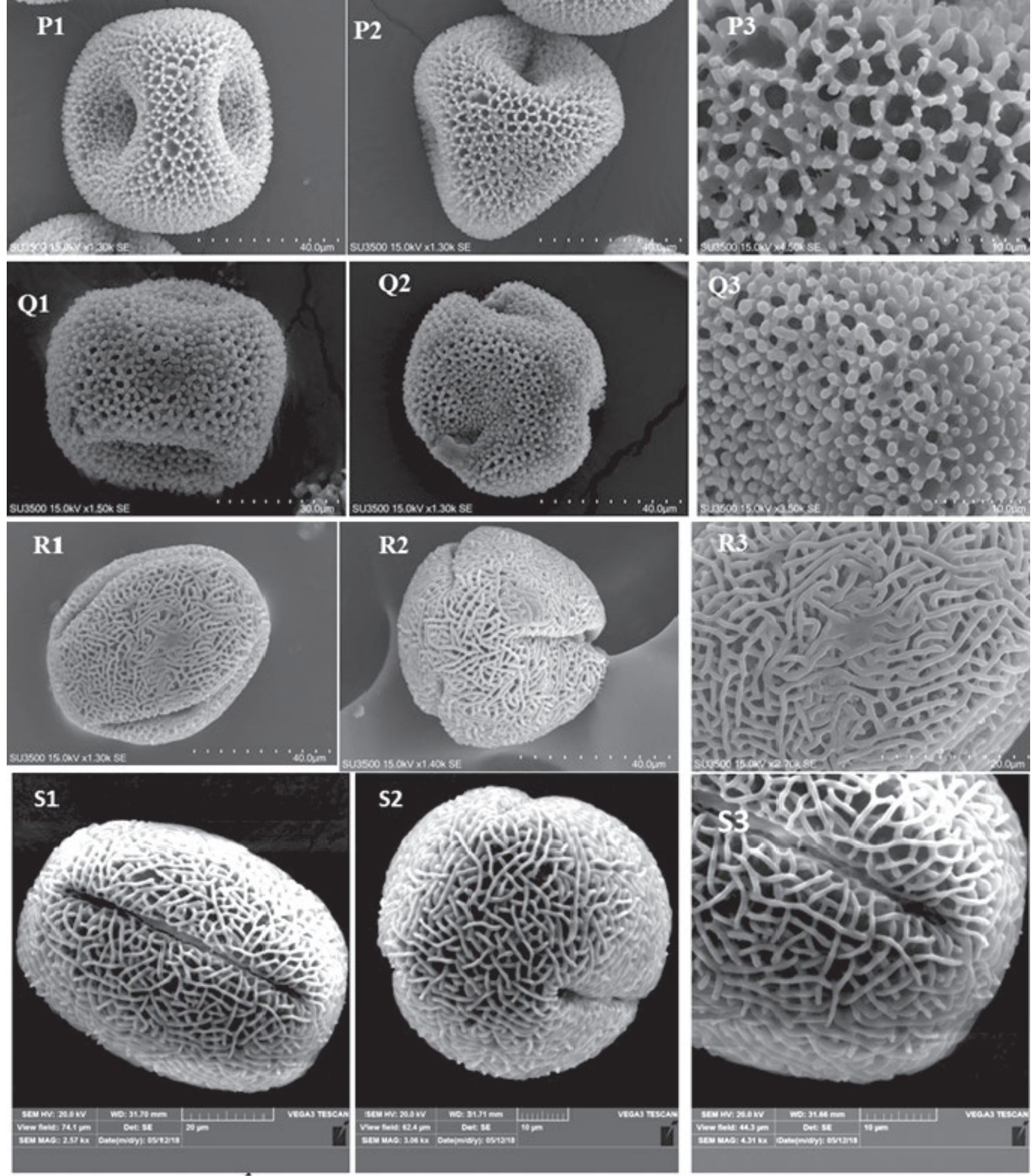

Figure 5. Pollen micrographs of Geranium species: P1-P3: G. robertianum, Q1-Q3: G. divaricatum, R1-R3: G. trilophum, S1-S3: G. mascatense; P1,Q1, R1, S1 - equatorial view; P2,Q2, R2, S2 - polar view; P3,Q3, R3, S3 -exine sculpture.

The discrepancy in the phylogenetic results obtained by different molecular markers may be the reason for unresolved species relationship in the genus Geranium. Evo- lutionary relationships between Geranium, Erodium, and are unclear because of the low support for the alternative topologies inferred from both markers ( $\operatorname{trnL}-F$ and $r b c L$ ) 

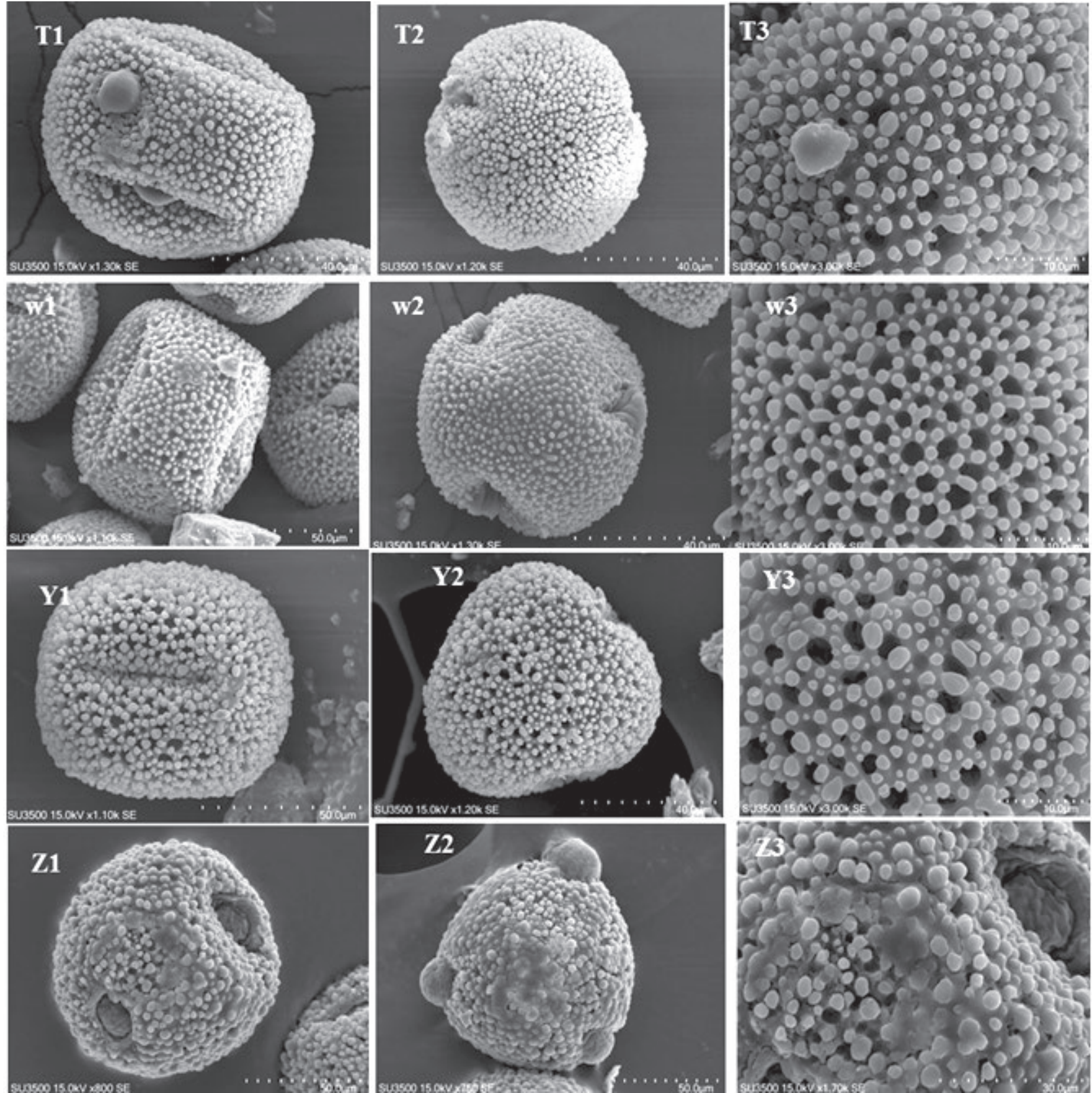

Figure 6. Pollen micrographs of Geranium species: T1-T3: G. kotschyi, W1-W3: G. linearilobum, Y1-Y3: G. tuberosum, Z1-Z3: G. platypetalum; T1,W1,Y1, Z1 - equatorial view; T2,W2,Y2, Z2 - polar view; T3,W3,Y3, Z3 -exine sculpture.

(Fiz et al. 2008). Inter-simple sequence repeat (ISSR) markers separated Geranium species (Esfandani-Bozchaloyi et al. 2018). The present study showed that pollen features are not capable of distinguishing different sections in Geranium. Two subgenera elements were mixed.

Nonetheless, we found and linked the diagnostic character (Pollen type III) of the Trilopha section. Future studies may incorporate phylogenetic data and chemotaxonomy methods to decipher genus and species level evolutionary relationship in Geranium. Linking the morphological and phylogenetic traits may further aid our apprehension in plant evolution and systematics (Cohen 2011).

In this research, we studied different palynological characteristics of the genus Geranium. Various characters were crucial for the taxonomic identification of the 

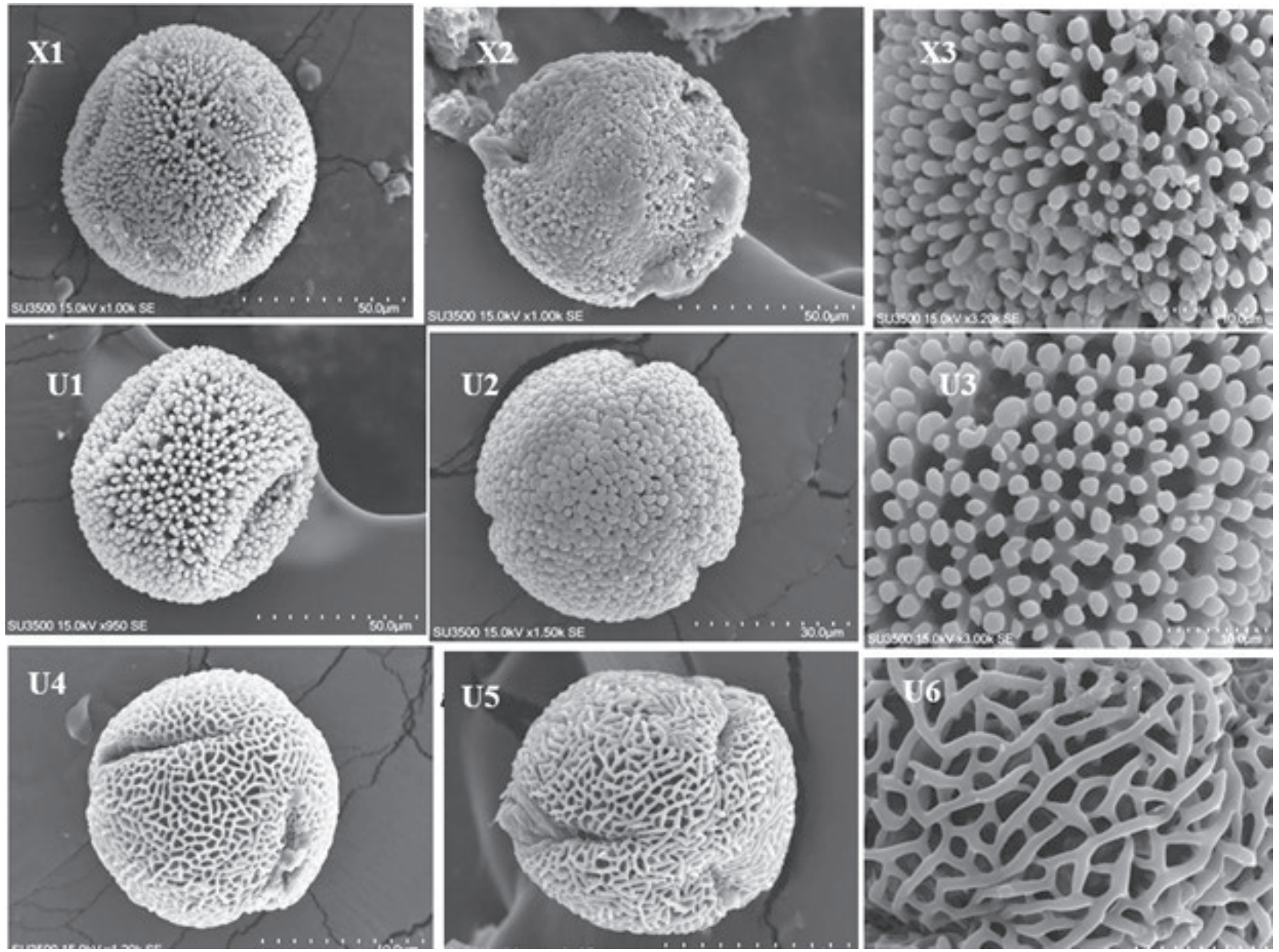

Figure 7. Pollen micrographs of Geranium species:X1-X3: G. ibericum, U1-U3: G. tuberosum, U4-U6: G. biuncinatum. X1,U1, U4 - equatorial view; X2, U2, U5- polar view; X3, U3, U6-exine sculpture.

species delimitations. This study could serve better to understand the pollen morphology of Geranium species in Iran. The pollen characters studied here were useful for the taxonomic identification of the species of the genus.

\section{ACKNOWLEDGEMENTS}

We would also thank Editor for his comments to improve the quality of the manuscript.

\section{REFERENCES}

Aedo C, Barbera P, Buira A. 2016. Taxonomic revision of Geranium sect. Trilopha (Geraniaceae). Syst Bot. 41:354-377.
Aedo C, Garcia M, Alarcon M, Aldasoro J, Navarro C. 2007. Taxonomic Revision of Geranium Subsect. Mediterranea (Geraniaceae). Syst Bot. 32:93-128.

Aedo C, Pando F. 2017. A distribution and taxonomic reference dataset of Geranium in the New World. Scientific Data. 4:170049.

Aedo C. 2001. Taxonomic revision of Geranium sect. Brasiliensia (Geraniaceae). Syst Bot. 26:205-215.

Aedo C. 2017. Taxonomic Revision of Geranium Sect. Ruberta and Unguiculata (Geraniaceae). Ann Missouri Bot Gard. 102:409-465.

Baser B, Firat M, Aziret A. 2016. The pollen morphology of Pelargonium endlicherianum and Pelargonium quercetorum (Geraniaceae) in Turkey. Phytokeys. 75:153-162.

Bortenschlager S. 1967. Vorlaufige Mitteilungen zur Pollen morphologie in der Geraniaceen und IHRE systematische Bedeutung. Grana Palynol. 7:400-468. 
Brodschneider R, Gratzer K, Kalcher-Sommersguter E, Heigl H, Auer W, Moosbeckhofer R. 2019. A citizen science supported study on seasonal diversity and monoflorality of pollen collected by honey bees in Austria. Sci Rep. 9(1):16633.

Cohen JI. 2011. A phylogenetic analysis of morphological and molecular characters of Lithospermum L. (Boraginaceae) and related taxa: evolutionary relationships and character evolution. Cladistics. 27(6):559580.

Deniz I, Cirpici A, Yildiz K. 2013. Palynological study of the Geranium (Geraniaceae) species from the Thrace region (Turkey-in-Europe). Phytologia Balcanica. 19:347-355.

El-Oqlah AA. 1983. Pollen morphology of the genus Erodium L'Hér. in the Middle East. Pollen et Spores. 25:383-394.

Erdtman G. 1960. The acetolysis method. A revised description. Svensk bot Tidskr. 39:561-564.

Fiz O, Vargas P, Alarcon M, Aedo C, García JL, Aldasoro JJ. 2008. Phylogeny and historical biogeography of Geraniaceae in relation to climate changes and pollination ecology. Syst Bot. 33:326-342.

Hammer O, Harper DAT, Ryan PD. 2001. PAST: paleontological statistics software package for education and data analysis. Palaeontol Electron. 4(1):1-9.

Hao K, Tian ZX, Wang ZC, Huang SQ. 2020. Pollen grain size associated with pollinator feeding strategy. Proc Biol Sci. 287:1933.

Hesse M, Halbritter H, Zetter R, Weber M, Buchner R, Frosch-Radivo A, Ulrich S. 2009. Pollen Terminology- An Illustrated Handbook. New York (NY): Springer. p.264.

Ilcim A, Dadandi MY, Cenet M. 2008. Morphological and palynological studies of Geranium tuberosum L. (Geraniaceae). J Appl Biol Sci. 2:69-73.

Image Tool Version 3.0. http://ddsdx.uthscsa.edu/dig/ itdesc.html.

Ji X, Hou C, Gao Y, Xue Y, Yan Y.,... Guo X. 2020. Metagenomic analysis of gut microbiota modulatory effects of jujube (Ziziphus jujuba Mill.) polysaccharides in a colorectal cancer mouse model. Food \& function, 11(1): 163-173.

Jurgens A, Witt T, Gottsberger G. 2012. Pollen grain size variation in Caryophylloideae: A mixed strategy for pollen deposition along styles with long stigmatic areas? Plant Syst Evol. 298:9-24.

Marcussen T, Meseguer AS. (2017). Species-level phylogeny, fruit evolution and diversification history of Geranium (Geraniaceae). Mol Phyl Evol. 110:134-149

Park SJ, Kim YS. 1997. A palynotaxonomic study of Asian Geranium L. Kor J Plant Tax. 27: 295-315.
Pathan AK, Bond J, Gaskin RE. 2010. Sample preparation for SEM of plant surfaces. Mater Today. 12:32-43.

Perveen A, Gaiser M. 1999. Pollen Flora of Pakistan. XV Geraniaceae. Turk J. Bot. 23: 263-269.

Podani J. 2000. Introduction to the exploration of multivariate data [English translation]. Leide (Netherlands): Backhuyes.

Shehata AA. 2008. Pollen morphology of Egyptian Geraniaceae: an assessment of taxonomic value. Int J Bot. 4:67-76.

Stafford PJ, Blackmore S. 1991. Geraniaceae. Rev Palaeobot Palynol. 69:49-78.

Sun S, Xu L, Zou Q, Wang G, Gorodkin J. 2021. BP4RNAseq: a babysitter package for retrospective and newly generated RNA-seq data analyses using both alignment-based and alignment-free quantification method. Bioinformatics, 37(9): 1319-1321.

Ullah F, Papini A, Shah SN, Zaman W, Sohail A, Iqbal M. 2019.Seed micromorphology and its taxonomic evidence in subfamily Alsinoideae (Caryophyllaceae). Microsc Res Tech. 82(3): 250-259.

Ullah F, Zaman W, Papini A, Zafar M, Nasar Shah S, Ahmad M, Saqib S, Gul S, Sohail A. 2019.Using multiple microscopic techniques for the comparative systematic of Spergula fallax and Spergula arvensis (Caryophyllaceae). Microsc Res Tech. 82(4):352-360.

Verhoeven RL, Venter HJT. 1986. Pollen morphology of Monsonia. S Afr J Bot. 52:361-368.

Verhoeven RL, Venter HJT. 1987. Pollen morphology of Erodium in southern Africa. S Afr J Bot. 53:279-283.

Wang X, Gao P, Liu Y, Li H, Lu F. 2020. Predicting Thermophilic Proteins by Machine Learning. Current Bioinformatics, 15(10): 493-502.

Weng M-L, Blazier JC, Govindu M, Jansen RK. 2014. Reconstruction of the ancestral plastid genome in Geraniaceae reveals a correlation between genome rearrangements, repeats, and nucleotide substitution rates. Mol Biol Evol. 31:645-659.

Yeo PF. 1984. Fruit-discharge-type in Geranium (Geraniaceae): its use in classification and its evolutionary implications. Bot J Linn Soc. 89:1-36.

Zou Q., Xing P., Wei L., Liu B. 2019. Gene2vec: gene subsequence embedding for prediction of mammalian $\mathrm{N}$ 6 -methyladenosine sites from mRNA. RNA (Cambridge), 25(2), 205-218. 